\title{
Seasonal variation of acute anterior uveitis: differences between HLA-B27 positive and HLA-B27 negative disease
}

\author{
ROLAND EBRINGER,' LAURINE WHITE,' RICHARD McCOY,' AND BRIAN TAIT ${ }^{2}$ \\ From the 'Department of Medicine, University of Melbourne, Austin Hospital; the ${ }^{1}$ Uveitis Research Clinic, \\ Royal Victorian Eye and Ear Hospital; and the ${ }^{2}$ Tissue Typing Laboratory, Royal Melbourne Hospital, \\ Australia
}

SUMMARY One hundred and seventy-five consecutive patients with acute anterior uveitis (AAU) were examined over a 24-month period. There was a significantly increased incidence of AAU during the months August to December $(p<0.05)$. This increase was confined predominantly to the HLA-B27 negative group of patients $(\mathrm{p}<0.01)$. There was no significant monthly difference in incidence between males and females, between patients with first or recurrent attacks, or between patients with and without arthritis. These results suggest that seasonal environmental factors may play a role in the production of HLA-B27 negative AAU.

The possibility that infection may trigger episodes of acute anterior uveitis (AAU) has been the subject of speculation and investigation for some years. In a small number of cases AAU may develop after an acute enteric infection with salmonella, ${ }^{1}$ shigella, ${ }^{2}$ or Yersinia enterocolitica. ${ }^{3}$ A genetic predisposition to the development of AAU is known. About half of all cases carry the tissue antigen HLA-B27. ${ }^{4}$ There is a strong association of AAU with various rheumatic diseases known as the seronegative spondylarthropathies, and most patients with these spondylarthropathies and those who develop AAU after enteric infection carry the HLA-B27 antigen.

Many ophthalmologists have hàd the impression that there are seasonal fluctuations in the incidence of AAU. Seasonal fluctuations in the frequency of a disease, particularly an acute inflammatory process such as AAU, suggest that an environmental factor may be involved in initiating or modulating the disease. It was therefore decided to examine the month of onset of all recorded episodes of AAU in a prospective, consecutive series of patients presenting over a 24 month period. The aim of the study was to determine if there was a seasonal pattern to AAU, and in particular to determine if there was any specific relationship with other variables such as

Correspondence to Dr R Ebringer, Department of Medicine, University of Melbourne, Austin Hospital, Heidelberg, Victoria 3084, Australia.
HLA tissue type, presence of arthritis, sex, and whether this was associated with first or recurrent attacks of AAU.

\section{Patients and methods}

Patient selection. One hundred and seventy-five consecutive patients with AAU were seen in the uveitis clinic over the 24-month period from April 1981 to March 1983. Patients with chronic uveitis (that is, those requiring more than three months of treatment) or patients with granulomatous or posterior uveitis were excluded from the study. Twenty-nine of these patients had earlier recorded and confirmed episodes of AAU with the month of onset available from the hospital records. Three patients had two episodes each during the study. In all 207 episodes were included. All patients were examined for HLA-A and B loci with the standard two-stage microlymphocytotoxicity assay.

Statistical analysis. Statistical analysis was carried out by $\chi^{2}$ with Yates's correction for small numbers. Analysis of the results was carried out in two ways. In the first instance a 12-cell $\chi^{2}$ analysis for incidence of AAU per month was examined. The null hypothesis was that there should be no excessive fluctuations in any month. This form of statistical analysis makes no attempt to examine any particular month but merely indicates whether there was a significant deviation 
from the expected monthly incidence. In the second analysis the patients were divided on the basis of HLA-B27 tissue type, sex, presence of arthritis or first or recurrent attacks, and again 12 cell $\chi^{2}$ tables were constructed.

\section{Results}

The results showed a statistically significant deviation from the expected observations when all AAU patients were examined (Fig. 1 ), $\chi^{2}=21 \cdot 3$, df $=11$, $p<0.05)$. When patients were divided on the basis of HLA-B27 tissue typing, the deviation was found to occur only in the B27 negative patients (Fig. 2), $\left(\chi^{2}=\right.$ $26 \cdot 0, \mathrm{df}=11, \mathrm{p}<0 \cdot 01)$. Inspection of the individual $\chi^{2}$ values in each cell of the table in both these studies showed that much of the largest contribution to the

No. of

episodes

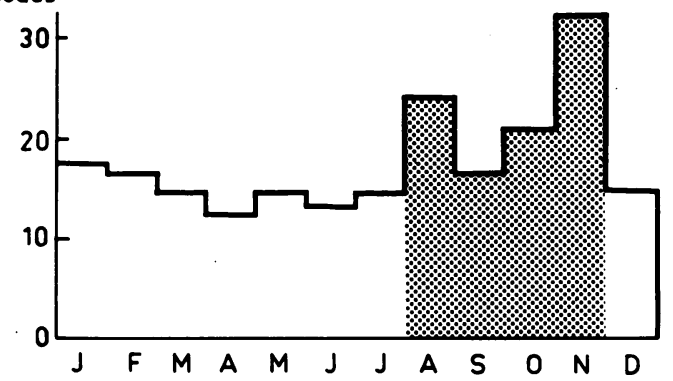

Fig. 1 Month of onset of 207 cases of $A A U$ in 175 patients.

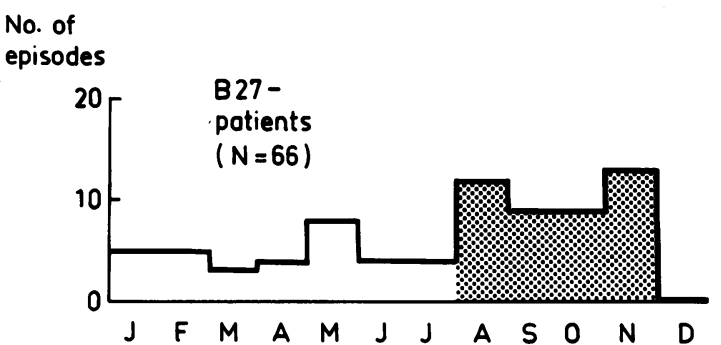

Fig. 2 Month of onset of AAU in HLA-B27 negative (B27-) patients.

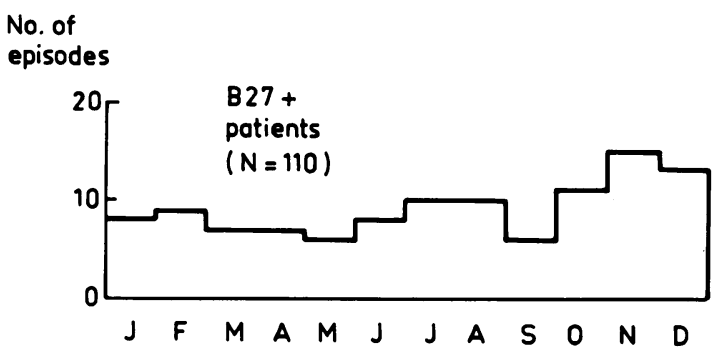

Fig. 3 Month of onset of AAU in HLA-B27 positive $(B 27+)$ patients. total came from the months of August to November, and particularly November itself. Although there was a slight increase in the incidence of HLA-B27 positive AAU in the four months from August to November inclusive (Fig. 3), there was no significant deviation from the expected results in this patient group. There was no statistically significant monthly difference in incidence observed in males or females, in patients with first or recurrent attacks, or in patients with and without arthritis.

\section{Discussion}

Our results showed a significant deviation of incidence of episodes of HLA-B27 negative AAU during the southern hemisphere spring months in a prospective group of patients studied in Melbourne. The hypothesis under test was not a priori related specifically to any particular month or group of months.

Although examination of the tables showed that the largest contribution to the $\chi^{2}$ value came from the spring months, it is not possible statistically to analyse these data further. To confirm this observation it would be necessary to examine a new series of patients, and investigate the specific hypothesis that there is a seasonal peak in HLA-B27 negative AAU during the spring months.

A seasonal change in hospital referral patterns appears unlikely to be the reason for the change. Firstly, there is no obvious specific reason for an increase in hospital referrals at that time such as holiday periods or unavailability of private ophthalmological treatment. Secondly, the observation that this increase in patients occurred in a subgroup of patients who have distinctive clinical and immunological features ${ }^{5-7}$ makes it more likely that the increase is a real observation and an important aetiological factor in the production of these diseases.

The most likely explanation for the seasonal fluctuation is the effect of a common environmental factor acting on the B27 negative subgroup of AAU patients. ${ }^{8}$ The possibilities include infective agents, dietary changes, or possibly seasonal allergic phenomena.

Apart from the small number of cases of AAU associated with Gram-negative enteric pathogens the evidence for infective factors in idiopathic AAU is preliminary. Recent reports have implicated faecal carriage Klebsiella pneumoniae with the development of AAU in patients with ankylosing spondylitis. ${ }^{9-10}$ We have recently observed a similar increase in AAU faecal carriage of klebsiella in a prospective series of unselected AAU patients. ${ }^{5}$ In the main, however, it has been HLA-B27 positive 
AAU patients who have been shown to have an association with Gram-negative bacteria.

An alternative explanation for the spring excess of AAU may be that the B27 negative subgroup of patients is affected by seasonal allergic factors. The spring months are the time of maximum pollen and grass related allergic phenomena. A recent report has suggested that serum IgE levels are raised in HLA-B27 negative AAU patients. ${ }^{7}$ These factors are currently under investigation in HLA-B27 negative associated AAU.

We are grateful to the many ophthalmologists, particularly Dr B Coote, who referred patients to us for investigation. These studies were supported by a grant from the OPSM Foundation, the Austin Hospital Research Foundation, and a research project grant from the Royal Victorian Eye and Ear Hospital. We are also grateful to Janet Finlayson, who typed the manuscript.

\section{References}

1 Saari KM, Vilpulla A, Lassus A, Leirisalo M, Saari R. Ocular inflammation in Reiter's disease after salmonella enteritis. $A m J$ Ophthalmol 1980; 90: 63-8.

2 Paronen I. Reiter's disease; a study of 344 cases observed in Finland. Acta Med Scand 1948; 131 (suppl 213): 1-114.

3 Saari KM, Laitinen O, Leirisalo M, Saari R. Ocular inflammation associated with Yersinia infection. Am J Ophihalmol 1980; 89: 84-95.

4 Brewerton DA. HL-A27 and acute anterior uveitis. Ann Rheum Dis 1975; 34(suppl 1): 33-5.

5 White L, McCoy R, Tait B, Ebringer R. A search for Gramnegative enteric micro-organisms in acute anterior uveitis: association of klebsiella with recent onset of disease, HLA-B27 and B7 CREG. Br J Ophthalmol in press.

6 McCoy R, White LF, Tait B, Ebringer R. Serum immunoglobulins in acute anterior uveitis. BrJ Ophthalmol in press.

7 Wakefield D, Easter J, Robinson P, Penny R. Immunological features of HLA-B27 anterior uveitis. Aust J Ophthalmol 1983; 11: $15-9$.

8 Barker DJP, Rose G. Epidemiology in medical practice. 2nd ed. London: Churchill-Livingstone, 1979: 70.

9 Ebringer R, Cawdell D, Ebringer A. Klebsiella pneumoniae and acute anterior uveitis in ankylosing spondylitis. Br Med J 1979; i: 383 .

10 Willshaw H, Eastmond C. Role of environmental factors in uveitis. Trans Ophthalmol Soc UK 1979; 99: 160-2. 